\title{
Entrelacs
}

Cinéma et audiovisuel

HS | 2008

Séries télé in/out

\section{Séries télévisées : la suite sur Internet}

\section{Rudy Morin}

\section{OpenEdition}

\section{Journals}

Édition électronique

URL : http://journals.openedition.org/entrelacs/271

DOI : 10.4000/entrelacs. 271

ISSN : 2261-5482

Éditeur

Éditions Téraèdre

\section{Édition imprimée}

Date de publication : 7 février 2008

ISSN : 1266-7188

Référence électronique

Rudy Morin, « Séries télévisées : la suite sur Internet », Entrelacs [En ligne], HS | 2008, mis en ligne le 01 août 2012, consulté le 05 mai 2019. URL : http://journals.openedition.org/entrelacs/271 ; DOI : 10.4000/entrelacs.271

Ce document a été généré automatiquement le 5 mai 2019.

Tous droits réservés 


\title{
Séries télévisées : la suite sur Internet
}

\author{
Rudy Morin
}

1 Les séries télévisées suscitent aujourd'hui auprès des téléspectateurs un enthousiasme tel que la majorité des chaînes de télévision, aux Etats-Unis comme en France, tendent à privilégier leur diffusion en prime time. Il suffit d'observer la succession des records d'audience réalisés par la diffusion de certaines séries télévisées pour apprécier l'ampleur du phénomène: 51,1 millions de téléspectateurs américains et 8,6 millions de téléspectateurs anglais regardaient le dernier épisode de Friends en 2004. En France, TF1 et M6 se partagent les séries à succès et obtiennent des audiences records grâce à des séries comme Prison Break, NCIS, ou encore Heroes en 2007, qui retiennent l'attention de plus de 6 millions de téléspectateurs et représentent parfois plus de $40 \%$ de part d'audience dès le lancement de la série. Sans toutefois tenter ici de relever toutes les raisons qui font le succès des séries télévisées, le phénomène peut s'expliquer notamment par une production de qualité, par la diversité des sujets traités et par le genre particulièrement efficace de la série-feuilleton, terme proposé par Martin Wincler (1999) dont la particularité est de mettre en haleine le téléspectateur qui voit évoluer l'intrigue sur une ou plusieurs saisons. Le budget de production des séries télévisées a également considérablement augmenté pour atteindre des sommets en 2004 avec le pilote (premier épisode) de la série Lost, les Disparus dont le budget avoisine les 10 millions de dollars. La qualité de la réalisation et du casting donne aux épisodes des allures de long métrage et les acteurs de séries télévisées profitent du phénomène et voient leur popularité rivaliser avec celle des plus grandes stars. La série télévisée est devenue par ailleurs le lieu de passage occasionnel pour de nombreuses célébrités sous la forme d'apparitions, afin de lui donner plus de crédit ou de relancer l'audience, à l'instar de la série Will And Grace qui a ainsi accueilli plus de deux cent guest stars (vedettes invitées). Le phénomène est mondial et touche également la communauté Internet qui s'organise sous forme de réseaux d'échanges (forums, réseaux de pair à pair, etc.) afin de diffuser légalement ou non les séries télévisées. Les internautes particulièrement séduits par une série n'hésiteront pas à se rendre sur les sites de communautés de fans, qui font vivre la série en dehors du petit écran, en partageant leurs points de vue, leurs commentaires, 
leurs satisfactions ou leurs déceptions, et s'essayant parfois à prédire la suite de l'intrigue, de la supposition la plus prévisible aux hypothèses les plus insensées.

\section{Le phénomène sur Internet}

2 Le constat de la prolifération et de la popularité croissante des séries télévisées n'est pas sans nous rappeler le phénomène similaire qui touchait les Etats-Unis et le Royaume-Uni dans les années 1970. Est-il réellement nécessaire de revenir sur le succès de la série Star Trek, diffusée pour la première fois en 1966, et qui sera suivie et commentée par les «trekkies» aujourd'hui encore. Nous pouvons également évoquer l'extraordinaire rassemblement que provoqua la diffusion du dernier épisode de M.A.S.H. en 1983, qui réunit plus de cent millions de téléspectateurs américains devant leur poste de télévision. Par ailleurs, la qualité des séries télévisées de l'époque n'a rien à envier à celles d'aujourd'hui si l'on se souvient de chef d'œuvres comme Le prisonnier de Patrick McGoohan à la fin des années 1960, ou plus tardivement Twin Peaks de David Lynch et Mark Frost. Ce qui nous permet de distinguer le phénomène tel qu'il a eu lieu dans les années 1960-1970 du phénomène actuel, c'est essentiellement le rôle des réseaux de distribution et de diffusion d'épisodes de séries télévisées sur Internet, dans la création, l'organisation et la visibilité des communautés de fans qui créent et alimentent l'aura des séries télévisées.

D'une part, le mode de consommation induit par Internet est l'un des facteurs de popularité des sites communautaires autour des séries télévisées ; leurs téléchargements, via les réseaux de pairs à pairs, est en forte croissance et il est possible aujourd'hui de trouver gratuitement tous les épisodes d'une série télévisée, tout en bénéficiant de l'immédiateté de leur mise en ligne, puisque ce sont les téléspectateurs eux-mêmes qui enregistrent l'épisode lors de sa première diffusion et le mette en partage dans les plus brefs délais. De plus, des équipes de sous-titrage amateur, appelé fansubs, contraction de fan et du diminutif anglais subtitle (sous-titre), prennent immédiatement le relais et proposent, parfois dès le lendemain, la traduction de l'épisode sous la forme d'un fichier de sous-titre à télécharger. Nous pouvons alors parler d'un véritable réseau de distribution alternatif de qualité qui offre, en termes de disponibilité et de rapidité, une solution plus performante que les modèles classiques de distribution de l'industrie de la vidéo en ligne.

4 Avec l'apparition sur le marché de technologies permettant la lecture de fichiers vidéo, différents modes de consommation se sont progressivement installés, du simple visionnage nomade à la projection privée en haute définition. En effet, il est possible de trouver en ligne l'épisode dont le format et la qualité ont été spécialement optimisés pour chacun des appareils de visionnage. On peut par exemple regarder le dernier épisode de " 24 Heures Chrono » sur sa console portable de dernière génération (Nintendo DS ou PSP), sur son baladeur numérique (Ipod vidéo ou autre Archos) et bientôt sur son téléphone portable. A l'inverse, voir un épisode directement en ligne, et sans le télécharger est également proposé par certains sites, peu enclins à respecter les droits de propriété et de diffusion. Parfois les épisodes sont tout simplement disponibles sur des services de partage de vidéo analogues aux célèbres plateformes Youtube et Dailymotion.

Ces différents modes de consommation jouent un rôle particulièrement important auprès des fans de séries télévisées. L'immédiateté et la disponibilité d'un épisode sur Internet 
créént un rendez-vous incontournable pour les fans tout autour du globe. Certains forums affichent même sur leur site le temps restant en secondes avant l'arrivée dudit épisode. La couverture de diffusion d'un épisode, et plus précisément les effets scénaristiques et l'intrigue de la série, concernent et passionnent alors des millions de téléspectateurs pratiquement au même moment, créant ainsi un véritable événement à chaque nouvelle sortie. C'est à cet instant que jaillissent sur Internet, et pas uniquement sur les sites officiels, quantités d'analyses, de spoilers (documents ou textes dévoilant une partie de l'intrigue), de résumés et de conjectures quant à la suite de la série, souvent illustrés de captures d'écran et accompagnés de commentaires. De plus, la portabilité de la vidéo permet aux fans de revoir à tout moment et dans n'importe quelle condition un épisode afin d'en avoir une nouvelle lecture ou pour la partager avec un autre fan tout aussi intéressé, ce qui rejoint Henry Jenkins (1992) lorsqu'il avançait « Fan reception can not and does not exist in isolation, but is always shaped through input from other fans " (La réception d'un fan ne peut exister de manière isolée, elle est toujours formée par l'intermédiaire d'un autre fan).

\section{Les domaines de fans comme espaces culturels}

6 La sortie d'une nouvelle série télévisée est accompagnée presque inévitablement d'une large campagne de promotion et d'information sur la toile. Outre les sites officiels déclinés en plusieurs langues, on peut compter parfois des dizaines de sites annexes qui participent au lancement de la série. L'histoire, les personnages, les résumés et les dates de sortie des épisodes, les analyses et les commentaires trouvent ainsi leur place sur les sites dédiés aux séries télévisées, sur l'encyclopédie en ligne Wikipédia ou encore sur le site des réseaux et des chaînes de télévision comme la FOX et HBO. Très souvent, la présence d'un forum sur ces sites permet d'ouvrir des fils de discussion et des débats autour de la série, donnant à chacun l'opportunité de découvrir ou d'approfondir l'univers et le scénario. Or ce sont tout particulièrement les sites créés par les fans, sites dits "non-officiels", qui nous permettent de repérer les principaux caractères de leur activité au sein des fandoms (contraction de fan et kingdom), domaines de prédilection d'un groupe de fans, qui transforment ces espaces culturels en véritables communautés actives de téléspectateurs. En effet, les fandoms, en plus de fournir les détails les plus pointus sur les arcannes de la série et de proposer quizz, livres d'or et divers téléchargements multimédia (fonds d'écran, bande-originale), se proposent comme des lieux d'échanges et de créations.

7 Les réalisateurs et l'équipe marketing de la série à succès Lost, les Disparus, récompensée à plusieurs reprises aux Emmy Awards et aux Golden Globe Awards, ont compris très rapidement l'intérêt qu'ils avaient à prendre au sérieux l'influence de l'activité des fans sur le succès d'une série. Jeffrey Jacob Abrams (2005), créateur de Lost, lançait lors d'une interview: "Internet a profondément modifié la manière dont nous regardons la télévision. Instantanément, des milliers de personnes réagissent et il se dégage très vite un consensus sur ce qu'ils aiment et n'aiment pas. [...] et il faudrait être fou pour ne pas écouter l'avis des fans. » Audelà de leur capacité à créer du mystère, à susciter le débat, voire à donner naissance chez les fans à des théories supposées éclairer le scénario, les créateurs de Lost ont conçu la série de telle manière qu'elle s'adresse autant au spectateur ordinaire qu'au fan invétéré, en offrant plusieurs niveaux de lecture et en ouvrant un espace de dialogue, ludique et interactif avec les fans. Dès le lancement de la série en 2004, des communautés 
de fans se créaient déjà sur les sites officiels, séduits tout simplement par l'épisode pilote, par la présence de Dominic Monaghan (Merry dans le Seigneur des Anneaux) ou par l'annonce de l'arrivée de David Fury et de Javier Grillo-Marxuach, scénaristes déjà suivis par des fans-clubs fidèles. Par la suite, les épisodes de Lost se sont constitués comme un véritable puzzle dans la compréhension et l'analyse de l'univers par les fans. Au fil des épisodes, chaque volet est devenu une aire de jeux pour le fan qui va chercher à découvrir et à interpréter tous les indices délibérément cachés par les scénaristes afin d'entretenir le mythe autour de la série. Qu'il s'agisse du mystère des numéros gagnants de Hurley à la loterie, du numéro du vol de l'avion, des symboles de Dharma Initiative, des hiéroglyphes sur le compteur, tout est finement élaboré pour entretenir l'activité des fans. Sur Internet, des groupes de fans spécialisés se sont organisés, les Whispers, acronyme pour We Hear Interesting Sounds Per Episode Replay (Nous entendons des bruits intéressants à chaque lecture) dont l'intérêt est d'élucider les murmures pratiquement inaudibles, ou encore les shippers (pour relationshippers) qui établissent des connexions entre tous les personnages de la série.

Suivre une série télévisée ne suffit plus, on veut pénétrer son univers, connaître les secrets des acteurs. Imaginer la suite n'est plus suffisant, on veut l'écrire. Voir les épisodes plusieurs fois n'est pas surprenant, on veut partager ses émotions, voir la réaction d'un autre fan, connaître les dialogues par cœur et trouver de nouveaux indices que la production aura glissés intentionnellement, ou non. L'univers d'une série suppose désormais son parallèle sur Internet, un espace de « liberté » pour les téléspectateurs qui participent pleinement à la construction d'une culture populaire dont les valeurs et les centres d'intérêt ne correspondent pas forcément à ceux des industries culturelles.

\section{Vers des espaces culturels populaires}

L'activité des fans de séries télévisées sur Internet dépasse largement le cadre d'un simple "dialogue » entre les créateurs d'une série et ses téléspectateurs. Le thème et l'univers abordés par une série télévisée, l'espace d'échange et d'interaction proposé par les sites spécialisés et la politique marketing (buzz, opérations marketing, conventions) sont incontestablement liés à l'ampleur du phénomène de socialisation des fans sur Internet. Cependant, certains indices nous permettent d'éclairer ce phénomène en écartant l'idée d'une manifestation récente, éphémère et contrainte. L'existence d'une terminologie très renseignée autour des séries télévisées témoigne de l'aspect communautaire des fans et l'analogie avec d'autres communautés nous incite à considérer ce phénomène dans le temps et de manière plus globale. Les fanfics (fanfictions) de séries télévisées sont des fictions écrites par les fans autour de l'univers ou des personnages. Issues de mouvements antérieurs comme celui des animefans (fans d'animations japonaises) et des fanzines, elles s'approprient l'œuvre originale, créant ainsi un véritable espace de création à l'intérieur même des fandoms. De plus, les fanfics crée un espace de liberté nécessaire au fan, un espace de réappropriation des mythes et des thèmes populaires abordés par les séries comme le souligne Henry Jenkins (1992) quand il écrit: "Fanfiction is a way of the culture repairing the damage done in a system where contemporary myths are owned by corporations instead of owned by folk» (La fan fiction est une manière pour la culture de réparer les dégâts commis dans un système où les mythes contemporains sont la propriété des entreprises au lieu d'être celle du peuple). 
Devant l'importance du phénomène, il convient de s'interroger sur la question de la consommation audiovisuelle aujourd'hui. Peut-on réellement parler de «fans", caractérisés généralement par l'excès, l'obsession et la vulnérabilité (Jenson Joli, 1992), lorsque l'on évoque les milliers d'inscrits et les millions de visiteurs qui participent à la construction de ces espaces culturels. Les domaines de fans ne se proposent-ils pas davantage comme un lieu de détournement et de réappropriation des produits culturels audiovisuels? Ces espaces de culture populaire ne tendent-ils pas à modifier nos modes de réception et d'appréhension des produits de l'industrie audiovisuelle?

\section{BIBLIOGRAPHIE}

SCHLERET Jean-Jacques, WINCKLER Martin, PETIT Christophe (1999). Les Séries Télé. Paris : Larousse (Histoire et Actualité). 407p. ISBN 2035111137.

JENKINS Henry (1992). Strangers No More, We Sing : Filking and the Social Construction of the Science Fiction Fan Community In LEWIS Lisa A. The Adoring Audience : fan culture and popular media. Londres : Lisa Lewis. 256p. ISBN 0415078202

JENKINS Henry (1992). Textual Poachers : Television Fans and Participatory Culture. (Studies in Culture and Communication). Jenkins Henry. 256p. ISBN 0415905729

ABRAMS Jeffrey Jacob (2005). In PORTER Lynette R. (2007). Unlocking the Meaning of Lost : An Unauthorized Guide. Napperville : Sourcebooks Inc. 304p. ISBN 1402207263

JENSON Joli (1992). Fandom as Pathology: The Consequences of Characterization In LEWIS Lisa A. The Adoring Audience : fan culture and popular media. Londres : Lisa Lewis. 256p. ISBN 041507820

\section{RÉSUMÉS}

Le succès manifeste des séries télévisées, diffusées sur les chaines américaines et européennes, ont très largement contribué à la mise en place de réseaux de distribution "parallèles " sur Internet. La disponibilité et l'immédiateté du téléchargement des épisodes, le jour suivant leur première diffusion, augmentent considérablement l'impact de leur sortie auprès des fans de la série. La couverture de diffusion d'un épisode, et plus particulièrement les effets scénaristiques de la série, concernent et passionnent alors des millions de téléspectateurs, de façon quasisimultanée, créant ainsi un véritable événement populaire. Sur Internet, les domaines de fans (ou fandoms) deviennent alors le lieu de rendez-vous pour des milliers d'internautes qui partagent alors commentaires, critiques et analyses sur les récents rebondissements de l'intrigue. Ces espaces communautaires constituent également des espaces de création où les fans se réapproprient l'univers de la série, s'essayant parfois à résoudre ses mystères, de la supposition la plus prévisible aux hypothèses les plus insensées, ou en imaginant des scénarii parallèles (fanfics), en marge du canon original.

L'univers d'une série suppose désormais son parallèle sur Internet, un espace de « liberté » pour les téléspectateurs qui participent pleinement à la construction d'une culture populaire. Il 
convient alors de saisir en quoi le mode de consommation de ce type de produit audiovisuel a changé et comment réagissent les créateurs et scénaristes afin de répondre à leur fans.

\section{AUTEUR}

\section{RUDY MORIN}

Doctorant LARA, Université Toulouse II Le Mirail 